\title{
PAS-Induced Potentiation of Cortical-Evoked Activity in the Dorsolateral Prefrontal Cortex
}

\author{
Tarek K Rajji, , 1,2, Yinming Sun 1,2, Reza Zomorrodi-Moghaddam ${ }^{1,2}$, Faranak Farzan', \\ Daniel M Blumberger ${ }^{1,2}$, Benoit $\mathbf{H}$ Mulsant', Paul B Fitzgerald ${ }^{3}$ and Zafiris J Daskalakis ${ }^{1,2}$ \\ 'Campbell Family Mental Health Research Institute at the Centre for Addiction and Mental Health, University of Toronto, Toronto, ON, \\ Canada; ${ }^{2}$ Temerty Centre for Therapeutic Brain Intervention at the Centre for Addiction and Mental Health, Toronto, ON, Canada; \\ ${ }^{3}$ Monash Alfred Psychiatry Research Centre, Alfred and Monash University Central Clinical School, Melbourne, VIC, Australia
}

\begin{abstract}
Neuroplasticity and long-term potentiation (LTP) in the dorsolateral prefrontal cortex (DLPFC) are considered important mechanisms in learning and memory, and their disruption may be related to the pathophysiology of several neuropsychiatric disorders. Paired associative stimulation (PAS) is a brain stimulation paradigm that produces enhanced activity in the human motor cortex that may be related to LTP. In a group of 15 healthy participants, we report on the potentiation of cortical-evoked activity in the human DLPFC using the combination of PAS and electroencephalography. In contrast, a PAS control condition did not result in potentiation in another group of nine healthy participants. We also demonstrate that PAS-induced potentiation of cortical-evoked activity is characterized by anatomical specificity that is largely confined to the site of stimulation. Finally, we show that PAS results in potentiation of $\theta$ - and $\gamma$-activity and $\theta$-phase- $\gamma$-amplitude coupling. These neurophysiological indices may be related to working memory, an important function of the DLPFC. To our knowledge, this is the first report of potentiation of cortical-evoked activity in the DLPFC. As this potentiation may be related to LTP, our findings provide a model through which neuroplasticity in health and disease states in the frontal cortex can be studied.

Neuropsychopharmacology (2013) 38, 2545-2552; doi:I0.1038/npp.2013.161; published online 24 July 2013
\end{abstract}

Keywords: dorsolateral prefrontal cortex; long-term potentiation; neuroplasticity; paired associative stimulation; transcranial magnetic stimulation

\section{INTRODUCTION}

The dorsolateral prefrontal cortex (DLPFC) is involved in a variety of executive functions, which have a role at various stages of memory formation and retrieval (Fuster, 2008). These functions include working memory (Goldman-Rakic, 1995), encoding information (Blumenfeld and Ranganath, 2006), and recall of information (Ranganath and D'Esposito, 2005). Although the neuronal mechanisms that underlie DLPFC functions are still not fully understood, Hebbian plasticity, particularly long-term potentiation (LTP), is thought to have a critical role. Reverberating circuits within the prefrontal cortex and between the prefrontal cortex and more posterior cortices provide the substrate for mutual and coincident excitations among neurons (Fuster, 2008; Petrides and Pandya, 2002). Such mutual and coincident excitations would facilitate LTP and the formation of associations during DLPFC functions (Brunel and Wang, 2001; Zipser et al, 1993).

\footnotetext{
*Correspondence: Dr TK Rajji, Campbell Family Mental Health Institute, Centre for Addiction and Mental Health, University of Toronto, 80 Workman Way, Room 6312, Toronto, ON M6I IH4, Canada, Tel: + | 416535 850।, Fax: + | 416537 |308,

E-mail: Tarek.Rajj@@camh.ca

Received II February 2013; revised 5 June 2013; accepted 7 June 2013; accepted article preview online 3 July 2013
}

Paired associative stimulation (PAS) is a brain stimulation paradigm that induces in vivo LTP-like increases in activity in the human cortex (Stefan et al, 2000). Recognizing that potentiation of cortical activity may not directly reflect the molecular and cellular changes that accompany synaptic LTP, PAS induces potentiation of cortical activity by simulating spike-timing-dependent plasticity paradigms (Markram et al, 1997). PAS consists of repeatedly delivering pairs of stimulations that occur contemporaneously in the cortex (eg, 180 pairs at $0.1 \mathrm{~Hz}$ for $30 \mathrm{~min}$ ). When applied to the motor cortex (M1), PAS consists of pairing electrical stimulation of the median nerve with- $25 \mathrm{~ms}$ latertranscranial magnetic stimulation (TMS) of M1. This interstimulus interval (ISI) of $25 \mathrm{~ms}$ is necessary for the two stimulations (peripheral nerve stimulation and TMS) to arrive simultaneously in M1 and result in potentiation of TMS-induced motor-evoked potential (MEP) in the corresponding muscle. MEP potentiation is the conventional index of M1 plasticity. This potentiation is proposed to be LTP-like given that it is characterized by associativity (ie, the need for a specific interval between the presynaptic inputs that arrive from different cortical regions), input specificity (ie, potentiation is focal and not distributed), and dependency on the N-methyl-D-aspartate (NMDA) receptor (Stefan et al, 2002).

No studies have been conducted to assess PAS-induced potentiation of cortical activity in the DLPFC. The challenge 
has been to identify a neurophysiological marker for the DLPFC that is analogous to MEP potentiation for M1. In this study, we investigated whether PAS results in the potentiation of activity in the DLPFC by combining TMS with electroencephalography (EEG) (Daskalakis et al, 2008) and using potentiation of cortical-evoked activity (CEA) as analogous to MEP potentiation. To assess whether PAS results in potentiation of CEA in the DLPFC, we combined peripheral nerve stimulation of the right median nerve with TMS to the left DLPFC using an ISI of $25 \mathrm{~ms}$. The rationale for stimulating the median nerve and using ISI of $25 \mathrm{~ms}$ as in PAS in M1 was based on a number of neuroanatomical and neurophysiological studies in rodents (Golmayo et al, 2003; Vaneden et al, 1992) and non-human primates (Goldman-Rakic, 1988; Petrides and Pandya, 1984). These studies are also supported by neurophysiological findings in humans. Stimulation of the median nerve produces a somatosensory-evoked potential over the contralateral frontal brain region of $24 \mathrm{~ms}$ latency (N24) (Larrea et al, 1992; Valeriani et al, 1997), with maximal amplitude at electrode F3, which overlies DLPFC (Larrea et al, 1992; Valeriani et al, 1998). This approach is consistent with several PAS studies in M1, where the ISI was chosen based on N20 peak of the somatosensory-evoked potential (Potter-Nerger et al, 2009; Ziemann et al, 2004). Using magnetoencephalography, activation of DLPFC has also been shown to occur in response to an oddball task that demands attention to median nerve stimulation (Huang et al, 2005a). Attention to median nerve stimulation, and hence DLPFC involvement is necessary (although not sufficient) in PAS (Stefan et al, 2004). These neurophysiological findings and the rich connections between the somatosensory and the prefrontal cortex suggested that PAS to the median nerve and DLPFC would result in potentiation of CEA in the DLPFC.

Cortical oscillations in the $\gamma$ - and $\theta$-band have an important role in the physiology of the DLPFC. For example, frontal midline $\theta$-power increases with increased working memory load (Gevins et al, 1997). $\gamma$-Oscillations in the frontal cortices have also been shown to increase linearly with an increase in working memory load (Howard et al, 2003) and to be associated with working memory deficits in patients with schizophrenia (Light et al, 2006). $\theta$-Power is also high in the frontal regions during visual and non-visual tasks (Voytek et al, 2010). Further, modulation of $\gamma$-amplitude by $\theta$-phase (' $\theta-\gamma$ coupling') has been associated with DLPFC function, particularly working memory (Canolty and Knight, 2010; Lisman and Idiart, 1995). Local neuronal networks that include recurrent perisomatic GABAergic inhibition onto excitatory and inhibitory neurons are essential for the generation of $\gamma$-oscillations (Bartos et al, 2007; Buzsaki and Wang, 2012b). Local and longer-range networks that include axoaxonic inhibition onto excitatory neurons, and excitatory input on apical dendrites of pyramidal neurons have also been implicated in the generation of $\theta$-oscillations (Buzsaki, 2002; Pignatelli et al, 2012). These findings imply that robust plasticity at these synapses is critical in the generation of $\theta$ - and $\gamma$-oscillations, and that the induction of plasticity in the prefrontal cortex would also manifest in an increase of $\gamma$ - and $\theta$-band EEG activity, as well as potentiation of $\theta-\gamma$ coupling.

Thus, the objective of this study was to evaluate PASinduced potentiation of CEA in the DLPFC. It was hypothesized that (1) PAS delivered to the DLPFC will result in potentiation of CEA, (2) potentiation of CEA will be localized to the site of stimulation (ie, DLPFC), and (3) potentiation of CEA would be band-specific, with potentiation limited to $\theta$ - and $\gamma$-bands frequencies, as well as $\theta-\gamma$ coupling.

\section{PARTICIPANTS AND METHODS}

\section{Participants}

Participants were aged $18-50$ years, as several studies pointed to changes in cortical neuroplasticity around 50 (eg, MullerDahlhaus et al, 2008); females and males; of all races and ethnicities; had no neuropsychiatric disorders; on no medications or substances that affect the CNS; right-handed to ensure homogeneity in hemisphere dominance; had no contraindication to TMS (Rossi et al, 2009) or MRI; and not pregnant. All participants provided written informed consent. In total, 15 participants received PAS and nine received a PAS control condition for comparison (see below).

\section{Localization of DLPFC}

Following published methods (Rusjan et al, 2010), we localized the left DLPFC using a T1-weighted MRI scan, the MRIcro/reg software, and the MINIBIRD system (Ascension Technologies, Burlington, VT). Stimulation of DLPFC was directed at the junction of the middle and anterior one-third of the middle frontal gyrus (Talairach coordinates $(x, y, z)=(-50,30,36))$.

\section{EEG Recording}

To measure CEA over the left DLPFC, we acquired EEG through a 64-channel Synamps 2 EEG system. All electrodes were referenced to an electrode positioned posterior to $\mathrm{Cz}$ electrode. EEG signals were recorded using DC and a lowpass filter of $100 \mathrm{~Hz}$ at $20 \mathrm{kHz}$ sampling rate, which was shown to avoid saturation of amplifiers and minimize TMSrelated artifact (Daskalakis et al, 2008).

\section{EEG Data Preprocessing}

EEG recordings were processed offline using MATLAB (The MathWorks, Natick, MA). EEG data were first downsampled from 20 to $1 \mathrm{KHz}$ and segmented with respect to TMS stimulus such that each epoch included 1000 -ms prestimulus baseline and 2000-ms post-stimulus activity. Epochs were baseline corrected with respect to the TMS-free prestimulus interval $(1000-110 \mathrm{~ms}$ before the TMS stimulus). The baseline-corrected post-TMS stimulus intervals $(25-1025 \mathrm{~ms})$, which were not contaminated by TMS artifact, were extracted and digitally filtered by using a zero-phase shift 1-120 Hz bandpass filter. Epochs were then visually inspected and trials were excluded if they had amplitudes larger than $\pm 100 \mu \mathrm{V}$; channels with flat EEG; or a pervasive artifact across a majority of channels (eg, head movement artifact). If a channel was exhibiting an artifact across most epochs, then the channel was extracted. The $60-\mathrm{Hz}$ powerline artifact was removed from each trial across all channels by using the Thomson F-test based on 
multitaper spectral estimate techniques (Percival and Walden, 1993). After power-line rejection, the artifact affected electrodes, and epochs were rejected automatically using related functions from EEGLAB toolbox.

Then, two rounds of independent component analysis (EEGLAB toolbox) were performed to reject TMS artifacts and eye-blink traces from the EEG data. Finally, an average signal was obtained from each electrode for every participant.

\section{Electromyography Recording}

Following established methods (Daskalakis et al, 2008), we recorded EMG from the right abductor pollicis brevis muscle and determined each participant's resting motor threshold (defined as the minimum stimulus intensity that elicits an MEP of more than $50 \mathrm{mV}$ in 5 of 10 trials). One hundred and twenty percent of the resting motor threshold was then used to begin the determination of the stimulus intensity of $1 \mathrm{mV}$ $\left(\mathrm{SI}_{1 \mathrm{mV}}\right)$, that is, the intensity of TMS that is needed to produce mean MEP amplitude of $1 \mathrm{mV}$ peak-to-peak at baseline over 20 TMS trials. The final $\mathrm{SI}_{1 \mathrm{mV}}$ was then used in all subsequent measurements of CEA in DLPFC.

\section{PAS and TMS-EEG in DLPFC}

We applied TMS to the left DLPFC as localized through neuronavigation (see above) using a 7-cm figure-of-eight coil and a Magstim 200 stimulator (The Magstim Company, Whitland, UK). To stimulate the left DLPFC, we delivered 100 single pulses of TMS at $\mathrm{SI}_{1 \mathrm{mV}}$ at $0.1 \mathrm{~Hz}$. During stimulation, we recorded CEA using Neuroscan (Compumedics, Charlotte, NC) from the EEG electrode that corresponded to the DLPFC and over which the TMS coil point-of-stimulation was placed. CEA was then defined as the rectified area under the curve (AUC) for these electrodes. The mean CEA generated from the 100 EEG recordings that correspond to the 100 TMS pulses was used in the final analyses. The time cutoffs used for calculating AUC were 50 and $275 \mathrm{~ms}$ after TMS stimulus. The $50 \mathrm{~ms}$ cutoff was chosen as it represents the earliest artifact-free data that can be recorded after TMS stimulus. The $275 \mathrm{~ms}$ cutoff was chosen as it represents the end of the last timewindow during which potentiation of CEA was still significant after PAS (Figure 1). Then, we delivered PAS, which consisted of 180 peripheral nerve stimulations delivered to the right median nerve, each paired with a single TMS pulse delivered to the left DLPFC. The median nerve stimulation preceded TMS by an ISI of $25 \mathrm{~ms}$. This ISI was designed to generate approximately synchronous arrival of both inputs in M1 and was reported to potentiate markedly the TMS-induced MEP following PAS in M1 (Stefan et al, 2000). Pairs of electrical stimuli and TMS were delivered at $0.1 \mathrm{~Hz}$ during a 30-min period to reach a total of 180 pairs. Electrical median nerve stimulation was delivered at $300 \%$ of the sensory threshold. The sensory threshold was determined as the lowest peripheral nerve stimulator intensity for which a participant perceives sensation in the median nerve sensory distribution. TMS was delivered at $\mathrm{SI}_{1 \mathrm{mV}}$. Attention during PAS has been previously demonstrated to have an important role in PAS in M1 (Stefan $e t$ al, 2004). To maintain the attention of participants during PAS, we asked participants to look at the hand being stimulated and count the total number of stimuli delivered over the 30 -min period of PAS. Although all participants were aware that the duration of PAS was several minutes, they were not

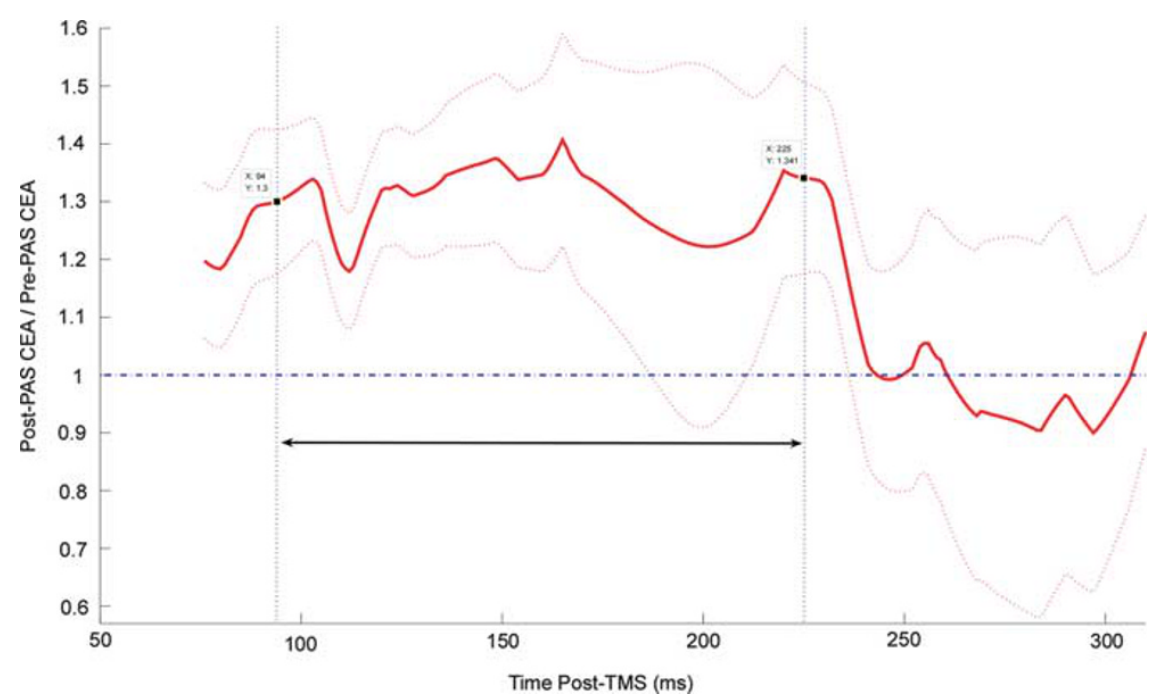

Figure I Time series of potentiation. This figure illustrates the time frame for overall potentiation following transcranial magnetic stimulation (TMS) pulse. Using the ratio of cortical-evoked activity (CEA) post-paired associative stimulation (PAS)/CEA pre-PAS for every I00-ms window after TMS with increments of I ms, we identified two time-points after TMS: the first is the earliest time-point of the first I00-ms window when the mean ratio becomes significantly larger than I (horizontal blue dashed line) and it occurred at $44 \mathrm{~ms}$ after TMS. This $100-\mathrm{ms}$ window is indicated by its midpoint (94 ms-left vertical blue dotted line) on the graph. The $50 \mathrm{~ms}$ cutoff that was used in calculating the area under the curve for overall potentiation and that was chosen to minimize TMS artifact falls within this window. The second time-point is the end of the last $100-\mathrm{ms}$ window during which the mean ratio remains significantly $>1$ and it occurred at $275 \mathrm{~ms}$ after TMS. This 100-ms window is also indicated by its midpoint (225 ms - right vertical blue dotted line) on the graph. This second time-point is what we used as the second cutoff in calculating the area under the curve for overall potentiation. Each data point (solid red line) represents the midpoint of a I00-ms window and corresponding median CEA ratio for that window. Dotted red lines: \pm I SEM. Black double-arrow: timeframe in which CEA ratios for all contiguously sliding 100-ms windows were significantly higher than I. 
aware of the frequency of the paired stimuli, nor of their total number. At Time 0,15 , and $30 \mathrm{~min}$ after PAS, we measured CEA according to the same methods described above for measuring CEA pre-PAS. These time points have been selected based on the time points at which maximum potentiation has been demonstrated in M1 (Frantseva et al, 2008). We then calculated PAS-induced potentiation using CEA at each of these three time-points using CEA ratio= CEA after PAS/CEA pre-PAS. As the post-PAS timing of maximum potentiation of CEA could vary among participants, we selected the maximum CEA ratio for each participant after PAS. Thereafter, we log-transformed these ratios to correct for skewed distribution, as a ratio cannot be smaller than 0 . We used one-sample $t$-test to test for significant potentiation.

\section{PAS Control Paradigm}

Following the same rationale, and as a control condition, we applied PAS with an ISI of $100 \mathrm{~ms}$ (PAS-C) in a separate group of nine healthy participants. An ISI of $100 \mathrm{~ms}$ has been shown not to result in potentiation of cortical activity in M1 (Stefan et al, 2000). Thereafter, using independent samples $t$-test, we compared mean maximum CEA ratios between the two groups: the group that received PAS with ISI of $25 \mathrm{~ms}$ and the group that received PAS-C with ISI of $100 \mathrm{~ms}$.

\section{Spatial Specificity of PAS-induced CEA Potentiation}

We assessed the spatial specificity of PAS-induced CEA potentiation at the target by comparing potentiation across all left (AF3, F3, F5, and F7) and right (AF4, F2, F4, F6, and F8) frontal electrodes, and global potentiation as captured from all electrodes to the reference value 1 using onesample $t$-test and Bonferroni corrections for multiple comparisons. We then compared potentiation across all left frontal electrodes to potentiation across all right frontal electrodes using paired samples $t$-test. This was conducted to evaluate the contention that CEA potentiation is input specific and, therefore, closely related to LTP.

\section{Frequency-Specific Potentiation}

Following previously published methods (Farzan et al, 2010), we used Matlab (The MathWorks, Natick, MA) and the EEGLAB toolbox to filter the EEG into the traditional frequency bands of $\delta(1-3.5 \mathrm{~Hz}), \theta(4-7 \mathrm{~Hz}), \alpha(8-12 \mathrm{~Hz})$, $\beta(12.5-28 \mathrm{~Hz})$, and $\gamma(30-50 \mathrm{~Hz})$ by using a zero-phase shift filter. Once the signal was filtered into the appropriate bands, we obtained AUC and CEA ratio following the same procedure as for overall CEA described above. We then assessed potentiation within each frequency band using one-sample $t$-test and Bonferroni corrections to correct for testing for each of the five bands.

\section{Effects on $\theta-\gamma$ Coupling}

We measured $\theta-\gamma$ coupling as a relationship between the $\theta$-phase and the $\gamma$-amplitude. We performed the analysis on the time-averaged file of each participant using Matlab by adapting previously published methods (Axmacher et al,
2010; Tort et al, 2010) and further developed in patients populations (Kirihara et al, 2012). We first filtered the signal into separate $\theta$ - and $\gamma$-waveforms with a zero-phase shift filter and then applied the Hilbert transform. Using the phase information of the $\theta$-wave, we sorted the corresponding $\gamma$-amplitudes into six bins (ie, $-180^{\circ}$ to $-120^{\circ},-120^{\circ}$ to $-60^{\circ},-60^{\circ}$ to $0^{\circ}, 0^{\circ}-60^{\circ}, 60^{\circ}-120^{\circ}, 120^{\circ}-180^{\circ}$ ), and then averaged them. As the angle values correspond to a cosine reference, zero degrees correspond to a peak of the waveform. To quantify coupling, we used an entropy-based modulation index (MI) (Tort et al, 2010):

$\mathrm{MI}=(\log (N)-H(P)] / \log (N)$

Where $N$ is the number of phase bins, $\log (N)$ represents the entropy of a uniform distribution, $P$ is the relative amplitude distribution sorted according to phase bins, and $H(P)$ is the entropy of the $P$ distribution, which is calculated as:

\section{$H(P)=-\sum_{j=1}^{N} P(j) \log [P(j)]$}

We calculated the relative amplitude distribution $P$ for each participant by dividing the amplitude of each phase by the sum of all amplitudes across bins. This maximum entropy for such a relative amplitude distribution happens when the amplitude is $1 / N$, which occurs when the distribution is uniform. As an increase in coupling represents an increase of order, higher coupling translates to lower entropy $H(P)$, which in turn results in a high MI value.

To assess whether coupling is present pre-PAS or after PAS, we compared the observed values to surrogate data. We generated surrogate data for each participant by maintaining the amplitude spectrum while randomizing the phase (Axmacher et al, 2010). A total of 200 iterations were carried out to generate an empirical distribution under the null hypothesis of no coupling. To assess whether PAS had any effect on coupling, we used the pre- and post-PAS and pre- and post-PAS-C MI distributions and performed a two-factor analysis of variance with Group (PAS vs PAS-C) as factor 1 and Time (Pre $v s$ Post) as factor 2. The choice of the post-PAS or PAS-C time-point for each participant was based on the maximum time of potentiation for the $\gamma$-frequency band.

\section{RESULTS}

\section{PAS-Induced Potentiation of Cortical Excitability}

We applied PAS to the left DLPFC of 15 right-handed healthy participants (mean age $=27.5 \pm 5.5 ; \mathrm{F}=5 ; 33.3 \%$; mean resting motor threshold $=41.2 \pm 5.9$ ). Participants' mean count of the paired stimulations (an index of attention known to be critical for the induction of potentiation of cortical excitability by PAS) was $182.3 \pm$ 5.7, not significantly different from $180\left(t_{14}=1.6, p=0.14\right)$, suggesting that participants were attending to PAS. Among these 15 participants, there was significant potentiation of CEA (mean CEA ratio $=1.34 \pm 0.44, t_{14}=3.40, p=0.004$, Cohen's $d=0.88)$.

We applied PAS-C with an ISI $=100 \mathrm{~ms}$ to the left DLPFC of nine right-handed healthy participants (mean age $=27.9 \pm 7.1 ; \quad \mathrm{F}=3 ; 33.3 \% ;$ mean resting motor threshold $=43.9 \pm 4.4)$. Participants' mean count of the paired stimulations was $175.6 \pm 12.4$, not significantly different from $\left.180\left(t_{8}=-1.08, p=0.31\right]\right)$, suggesting that 


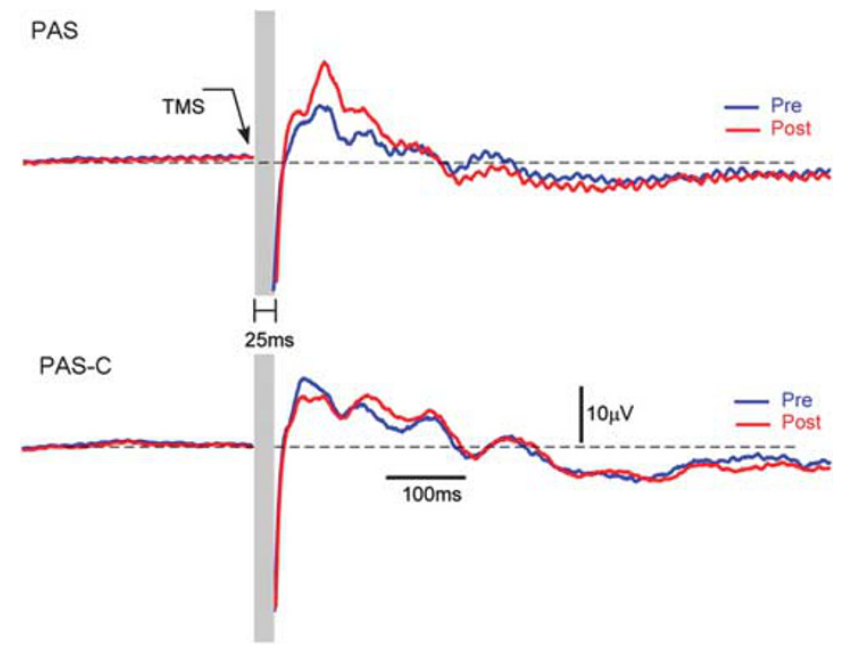

Figure 2 Time series of transcranial magnetic stimulation (TMS)-evoked response potential after paired associative stimulation (PAS) and paired associative stimulation-control condition (PAS-C). The upper panel illustrates the potentiation of the TMS-evoked response potential after PAS (Post) compared with pre-PAS (Pre). The lower panel illustrates the absence of this potentiation following the control condition, PAS-C.

participants were attending to PAS-C. Among these nine participants, there was no significant potentiation of CEA (mean CEA ratio $=0.90 \pm 0.22, t_{8}=-1.59, p=0.15$ ).

Comparing the two groups (PAS vs PAS-C), participants did not differ in age $\left(t_{22}=0.14, p=0.89\right)$, sex $\left(\chi_{1}^{2}=0.00\right.$, $p=1.0)$, or resting motor threshold $\left(t_{22}=1.16, p=0.26\right)$. However, the group that received PAS experienced significant potentiation in CEA compared with the group that received PAS-C $\left(t_{22}=3.33, p=0.003\right.$, Cohen's $\left.d=1.4\right)$ (Figures 2 and 3). CEA changes for each participant at all time-points $(0,15$, and $30 \mathrm{~min})$ after PAS or PAS-C are illustrated in Supplementary Figure S1.

\section{Spatial Specificity of PAS-Induced Potentiation of CEA}

We found that potentiation at the target electrode (as reported above) and across left frontal electrodes was significantly $>1\left(t_{14}=5.26, p=0.000\right.$; Cohen's $\left.d=1.36\right)$, but not across right frontal electrodes $\left(t_{14}=2.0, p=0.19\right)$, or globally $\left(t_{14}=0.81, p=1.0\right)$ (Figure 4$)$. We also found that potentiation across the left frontal electrodes was significantly higher than that across right frontal electrodes $\left(t_{14}=2.69, p=0.018\right)$.

\section{Frequency-Specific Potentiation of Cortical Excitability}

With respect to frequency-specific potentiation, as expected there was potentiation within the $\gamma\left(t_{14}=4.00, p=0.005\right.$, Cohen's $d=1.03)$ and $\theta\left(t_{14}=3.42, p=0.02\right.$, Cohen's $d=0.88)$ bands but also within $\delta\left(t_{14}=3.28, p=0.015\right.$, Cohen's $d=0.91)$. There was no potentiation within the $\alpha$ $\left(t_{14}=1.75, p=0.5\right)$ or $\beta\left(t_{14}=0.32, p=1.0\right)$ frequency bands.

\section{Effects on $\boldsymbol{\theta}-\boldsymbol{\gamma}$ Coupling}

Compared with surrogate data, we found that there was significant coupling in response to TMS both pre- and after PAS or PAS-C $(p$ 's $<0.005)$. We also found that there was a

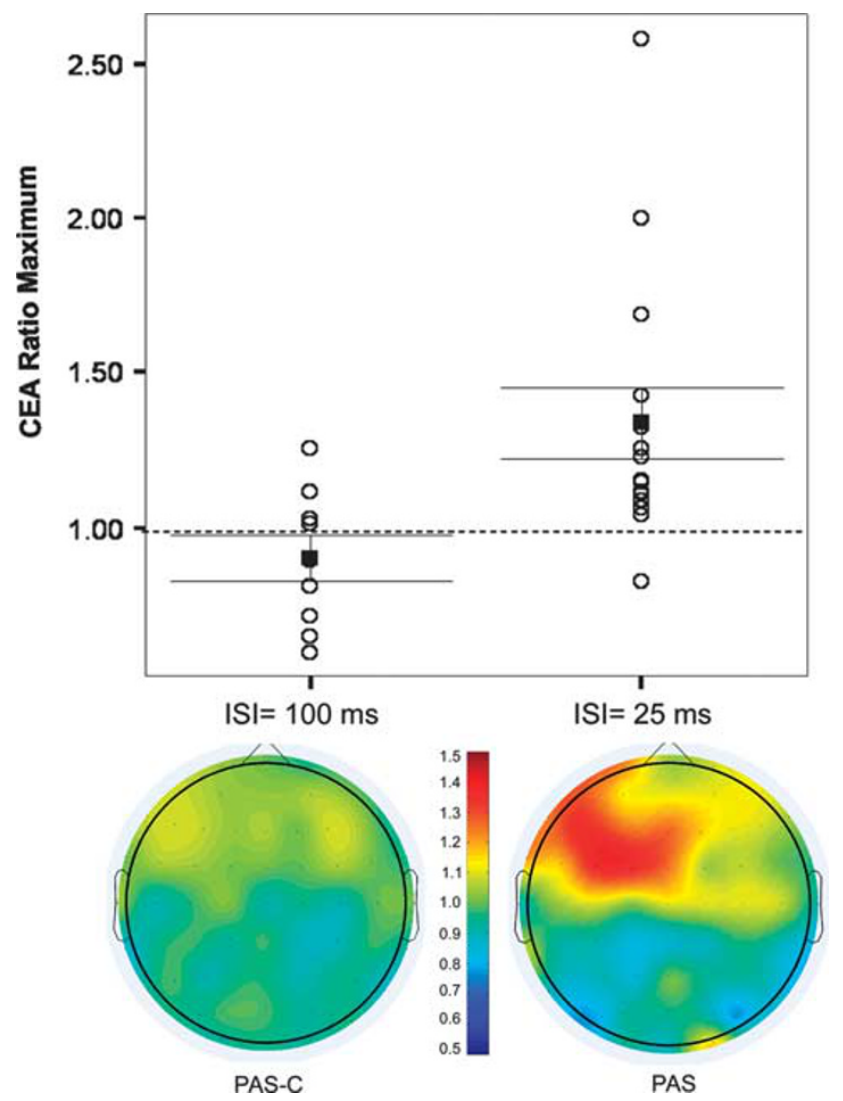

Figure 3 Paired associative stimulation (PAS)-induced potentiation of cortical excitability: 25 vs 100 ms interstimulus interval (ISI). The upper panel illustrates that PAS with an ISI of 25 ms-but not $100 \mathrm{~ms}$ (paired associative stimulation - control condition (PAS-C)) - between the peripheral nerve stimulation and transcranial magnetic stimulation (TMS) results in the potentiation of cortical excitability as indexed by maximum cortical-evoked activity (CEA) ratio significantly $>$ I (black line). The lower panel demonstrates the average topoplots of PAS and PAS-C. Circles: individual participants' data. Error bars: \pm I SEM.

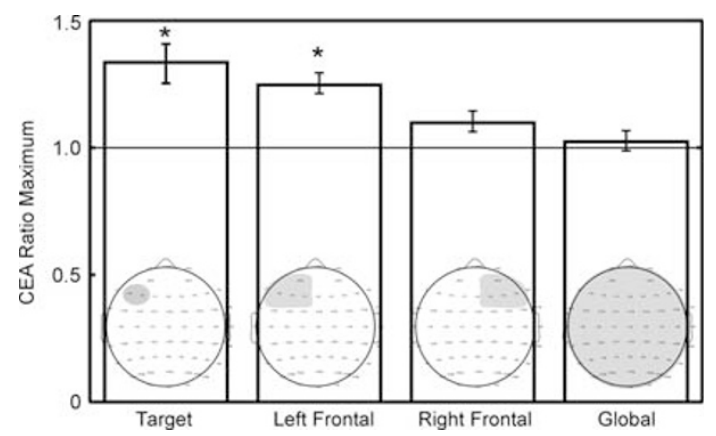

Figure 4 Localization of potentiation. This figure illustrates that paired associative stimulation (PAS) results in significant potentiation $\left(^{*}\right)$ at the site of transcranial magnetic stimulation (TMS) stimulation over the left dorsolateral prefrontal cortex (DLPFC) (Target) and across the left frontal region (Left frontal) when mean cortical-evoked activity (CEA) ratio is compared with the reference value of I (black line) $\left(p^{\prime} s<0.005\right)$. In contrast, no significant potentiation is observed in the contralateral frontal region (Right frontal) or over all electrodes (Global). Error bars: \pm I SEM.

significant interaction between Group (PAS vs PAS-C) and Time (Pre vs Post): $\mathrm{F}_{1,44}=5.53, p=0.023$. Post hoc analyses revealed that MI was not significantly different between 


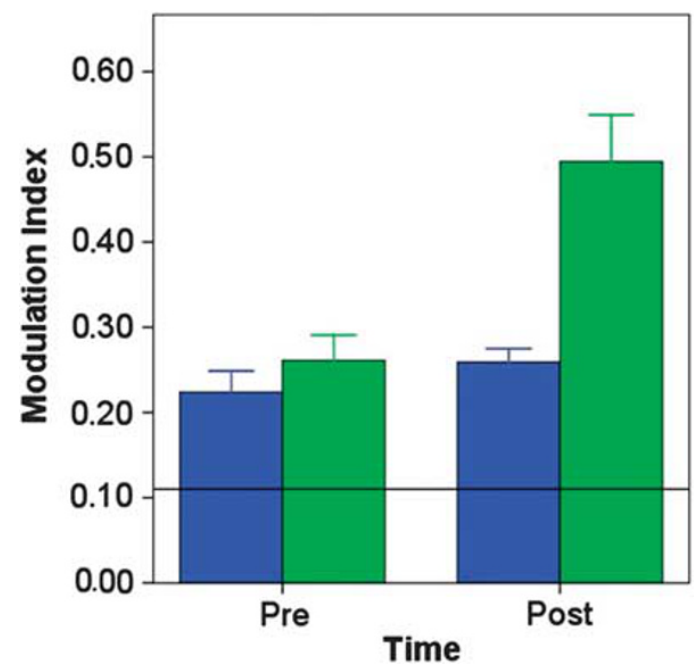

Figure 5 Paired associative stimulation (PAS) effects on $\theta-\gamma$ coupling. This figure illustrates that PAS and not paired associative stimulationcontrol condition (PAS-C) increases the modulation index (MI) of $\theta$-phase $\gamma$-amplitude coupling $(\theta-\gamma$ coupling) in the dorsolateral prefrontal cortex (DLPFC). $\mathrm{Ml}$ in response to transcranial magnetic stimulation (TMS) is significantly greater than $\mathrm{Ml}$ of surrogate data (black line) before and after PAS and PAS-C. However, following PAS and not PAS-C, MI significantly increases further. Error bars: \pm I SEM.

the two groups pre-PAS/PAS-C $\left(t_{22}=0.87, p=0.4\right)$, but significantly higher in the PAS group compared with the PAS-C group after PAS/PAS-C $\left(t_{22}=3.24, p=0.004\right.$, Cohen's $d=1.29$ ). They also revealed that post-PAS MI was significantly higher than pre-PAS MI (paired samples $t_{14}=4.53$, $p<0.001$, Cohen's $d=2.18$ ); in contrast, post-PAS-C MI was not significantly different than pre-PAS-C MI (paired samples $t_{9}=1.47, p=0.18$ ) (Figure 5). MI values for each participant at all time-points pre- and after PAS or PAS-C $(0,15$, and $30 \mathrm{~min}$ ) are illustrated in Supplementary Figure S2.

\section{DISCUSSION}

In this report, we present the first demonstration of PAS-a brain stimulation paradigm that simulates synaptic spiketiming-dependent plasticity (Markram et al, 1997)induces potentiation of CEA in the human DLPFC. We also report that this potentiation is associative in nature as it depends on an optimal ISI between the two paired inputs; that it is input-specific as it is localized to the side of stimulation; and that it is associated with robust potentiation within the $\gamma$ - and $\theta$-frequency bands, as well as their coupling.

EEG indexes cortical activity that is associated closely with synaptic activity (Buzsaki et al, 2012a). In turn, TMS produces trans-synaptic activation of the cortex (Rothwell, 1991). Thus, our findings suggest that weak synapses that are not stimulated in response to single-pulse TMS before PAS are strengthened by PAS. This strengthening of synapses (ie, LTP) translates into potentiation of CEA as captured by EEG. PAS-induced CEA potentiation may be related to LTP for several reasons. First, the associative property of PAS-induced CEA potentiation is analogous to the associative nature of synaptic LTP (Barrionuevo and Brown, 1983). Second, the input-specificity of PAS-induced CEA potentiation-it was demonstrated in the left frontal cortex and not in other cortical regions-is analogous to the input-specific nature of synaptic LTP (Nishiyama et al, 2000). Finally, the fact that PAS resulted in potentiation within the $\gamma$ - and $\theta$-frequency bands is also consistent with a synaptic effect. $\gamma$-Oscillations depend on local recurrent perisomatic inhibition onto excitatory and inhibitory neurons (Bartos et al, 2007; Buzsaki and Wang, 2012b). $\theta$-Oscillations are also dependent on functional local and longer-range networks (Buzsaki, 2002; Pignatelli et al, 2012). Thus, potentiation within these two frequencies suggests that PAS activates new synapses that are elements of such networks.

Modulation of $\theta$ - and $\gamma$-oscillations and their coupling are proposed as neurophysiological indices of DLPFC function, especially working memory (Canolty and Knight, 2010; Lisman and Idiart, 1995). In particular, it is proposed that each $\gamma$-oscillation that is coupled to a specific phase of the $\theta$-cycle represents specific information. Consequently, with each $\theta$-cycle this information is reactivated as the $\gamma$-oscillations are reactivated. Moreover, the order of various $\gamma$-oscillations represents the ordering of different information, which is an important aspect of working memory. The relationship between $\theta-\gamma$ coupling and working memory has been demonstrated in humans using intracranial (Axmacher et al, 2010) and scalp (Park et al, 2013) EEG recordings. Thus, our finding of PAS-induced potentiation of $\theta-\gamma$ coupling suggests that PAS activates neuronal networks and synapses that are likely to be activated when the DLPFC is behaviorally engaged in working memory task (Gevins et al, 1997; Howard et al, 2003). It also suggests that PAS could enhance working memory through the enhancement of $\theta-\gamma$ coupling in populations with impairments in working memory and associated neurophysiologic markers (Light et al, 2006). Notwithstanding the relationship between frontal $\gamma$ - and $\theta$-oscillations and working memory, these oscillations have been associated with a broad range of cognitive functions. These functions span from simple perception such as auditory processing (Galambos et al, 1981) to higherorder cognition such as executive functions and social cognition (Uhlhaas and Singer, 2006). Thus, the implications of our findings may be limited to non-working memory functions or could span across various levels of cognition.

Other neurophysiological paradigms have been developed to assess neuroplasticity and LTP-like activity in the human cortex. Simulating in vitro high-frequency stimulation, sensory stimulation plasticity paradigms assess LTP-like activity in the auditory or visual cortices through the repetitive delivery of auditory or visual stimuli in combination with the measurement of auditory or visual-evoked potentials (Clapp et al, 2012). $\theta$-Burst stimulation is a TMSbased paradigm that simulates in vitro $\theta$-burst stimulation in generating LTP-like activity. It has mainly been used to assess plasticity in M1 (Huang et al, 2005b). Transcranial direct current stimulation is another brain stimulation method that also has been used to assess plasticity in M1 (Nitsche and Paulus, 2000). Simulating spike-timing-dependent plasticity, our study demonstrates LTP-like activity directly from the DLPFC. Whether or not our findings relate to other forms of plasticity (eg, sensory- or $\theta$-burst stimulation) remains to be determined.

Our study has some limitations. One limitation is that we used the same ISI of $25 \mathrm{~ms}$ as the one used in M1. Although 
our findings confirm CEA potentiation, it is possible that other ISIs can produce more robust potentiation given that some projections from the somatosensory cortex to the DLPFC are multisynaptic (Miller and Cohen, 2001). Other ISIs could also result in less variability in the timing of CEA potentiation. Systematic evaluations of various ISIs could be investigated in future studies as well as the use of individualized N24 to determine ISI for each participant. Determining individualized ISIs is also consistent with the literature on PAS in M1, and could result not only in less variability with respect to the timing but also in the degree of potentiation, which is also observed in M1 (Muller-Dahlhaus et al, 2008; Stefan et al, 2004).

Another limitation is that, whereas our findings support an LTP-like nature of CEA potentiation, pharmacological studies need to confirm its relationship to NMDA as it has been demonstrated with PAS in M1 (Stefan et al, 2002).

In conclusion, using PAS and TMS-EEG, we report PASinduced potentiation of CEA in the DLPFC. Such potentiation was charactericized by anatomical, temporal, and frequency specificities, and may be related to LTP-like activity in this brain region. Future studies combining this paradigm with neurocognitive tasks and/or functional imaging, and contrasting it with other plasticity paradigms may further elucidate the role of DLPFC plasticity in cognition or help to identify future illness and treatmentrelated biomarkers for severe neurological and psychiatric disorders.

\section{FUNDING AND DISCLOSURE}

Dr Rajji received research support from Brain and Behavior Research Foundation (previously known as NARSAD), Canadian Foundation for Innovation, Canadian Institutes of Health Research (CIHR), Ontario Ministry of Health and Long-Term Care, Ontario Ministry of Research and Innovation, the US National Institute of Health (NIH), and the W Garfield Weston Foundation; Mr Sun received research support from CIHR; Dr Farzan received research support from CIHR; and Dr Blumberger received research support from Brain and Behavior Research Foundation (previously known as NARSAD), CIHR, and Brainsway for an investigator-initiated study. Dr Mulsant received research support from CIHR, NIH, Bristol-Myers Squibb (medications for an NIH-funded clinical trial), and Pfizer (medications for an NIH-funded clinical trial). He directly owns stocks of General Electric $(<\$ 5000)$. Within the past 5 years, he has also received some grant support from Eli Lilly (medications for an NIH-funded clinical trial) and Janssen, and some travel support from Roche. Dr Fitzgerald received in the past 2 years equipment for research from Brainsway, Medtronic, and MagVenture A/S, and funding for research from Cervel Neurotech. Dr Daskalakis received external funding through Neuronetics and Brainsway, Aspect Medical, and travel allowance through Pfizer and Merck, he received speaker funding through Sepracor, and also served on the advisory board for Hoffman-La Roche Limited. Dr Daskalakis received research support from the Brain and Behavior Research Foundation, CIHR, the Grant Family through the Centre for Addiction and Mental Health Foundation, and Ontario Mental Health Foundation. None of the above result in a potential conflict of interest.

\section{ACKNOWLEDGEMENTS}

We thank the Campbell Family and the Temerty Family for supporting this research at the Center for Addiction and Mental Health.

\section{REFERENCES}

Axmacher N, Henseler MM, Jensen O, Weinreich I, Elger CE, Fell J (2010). Cross-frequency coupling supports multi-item working memory in the human hippocampus. Proc Natl Acad Sci USA 107: 3228-3233.

Barrionuevo G, Brown TH (1983). Associative long-term potentiation in hippocampla slices. Proc Natl Acad Sci USA 80: 7347-7351.

Bartos M, Vida I, Jonas P (2007). Synaptic mechanisms of synchronized gamma oscillations in inhibitory interneuron networks. Nat Rev Neurosci 8: 45-56.

Blumenfeld RS, Ranganath C (2006). Dorsolateral prefrontal cortex promotes long-term memory formation through its role in working memory organization. J Neurosci 26: 916-925.

Brunel N, Wang XJ (2001). Effects of neuromodulation in a cortical network model of object working memory dominated by recurrent inhibition. J Comput Neurosci 11: 63-85.

Buzsaki G (2002). Theta oscillations in the hippocampus. Neuron 325-340.

Buzsaki G, Anastassiou CA, Koch C (2012a). The origin of extracellular fields and currents-EEG, ECoG, LFP and spikes. Nat Rev Neurosci 13: 407-420.

Buzsaki G, Wang XJ (2012b). Mechanisms of gamma oscillations. Annu Rev Neurosc (Palo Alto, CA) 203-225.

Canolty RT, Knight RT (2010). The functional role of crossfrequency coupling. Trends Cogn Sci 14: 506-515.

Clapp WC, Hamm JP, Kirk IJ, Teyler TJ (2012). Translating longterm potentiation from animals to humans: a novel method for noninvasive assessment of cortical plasticity. Biol Psychiatry 71: 496-502.

Daskalakis ZJ, Farzan F, Barr MS, Maller JJ, Chen R, Fitzgerald PB (2008). Long-interval cortical inhibition from the dorsolateral prefrontal cortex: a TMS-EEG study. Neuropsychopharmacology 33: $2860-2869$.

Farzan F, Barr MS, Levinson AJ, Chen R, Wong W, Fitzgerald PB et al (2010). Evidence for gamma inhibition deficits in the dorsolateral prefrontal cortex of patients with schizophrenia. Brain 133: 1505-1514.

Frantseva MV, Fitzgerald PB, Chen R, Moller B, Daigle M, Daskalakis ZJ (2008). Evidence for impaired long-term potentiation in schizophrenia and its relationship to motor skill leaning. Cereb Cortex 18: 990-996.

Fuster JM (2008). The Prefrontal Cortex. Academic Press: London, UK.

Galambos R, Makeig S, Talmachoff PJ (1981). A 40-Hz auditory potential from the human scalp. Proc Natl Acad Sci USA 78: 2643-2647.

Gevins A, Smith ME, McEvoy L, Yu D (1997). High-resolution EEG mapping of cortical activation related to working memory: effects of task difficulty, type of processing, and practice. Cereb Cortex 7: 374-385.

Goldman-Rakic PS (1988). Topography of cognition: parallel distributed networks in primate association cortex. Ann Rev Neurosci 11: 137-156.

Goldman-Rakic PS (1995). Cellular basis of working-memory. Neuron 14: 477-485.

Golmayo L, Nunez A, Zaborszky L (2003). Electrophysiological evidence for the existence of a posterior cortical-prefrontalbasal forebrain circuitry in modulating sensory responses in visual and somatosensory rat cortical areas. Neuroscience 119: 597-609. 
Howard MW, Rizzuto DS, Caplan JB, Madsen JR, Lisman J, Aschenbrenner-Scheibe R et al (2003). Gamma oscillations correlate with working memory load in humans. Cereb Cortex 13: 1369-1374.

Huang MX, Lee RR, Miller GA, Thoma RJ, Hanlon FM, Paulson KM et al (2005a). A parietal-frontal network studied by somatosensory oddball MEG responses, and its cross-modal consistency. NeuroImage 28: 99-114.

Huang YZ, Edwards MJ, Rounis E, Bhatia KP, Rothwell JC (2005b). Theta burst stimulation of the human motor cortex. Neuron 45: 201-206.

Kirihara K, Rissling AJ, Swerdlow NR, Braff DL, Light GA (2012). Hierarchical organization of gamma and theta oscillatory dynamics in schizophrenia. Biol Psychiatry 71: 873-880.

Larrea LG, Bastuji H, Mauguiere F (1992). Unmasking of cortical SEP components by changes in stimulus rate-a topographic study. Electroencephalogr Clin Neurophysiol 84: 71-83.

Light GA, Hsu JL, Hsieh MH, Meyer-Gomes K, Sprock J, Swerdlow NR et al (2006). Gamma band oscillations reveal neural network cortical coherence dysfunction in schizophrenia patients. Biol Psychiatry 60: 1231-1240.

Lisman JE, Idiart MAP (1995). Storage of $7+/-2$ short-term memories in oscillatory subcycles. Science 267: 1512-1515.

Markram H, Lubke J, Frotscher M, Sakmann B (1997). Regulation of synaptic efficacy by coincidence of postsynaptic APs and EPSPs. Science 275: 213-215.

Miller EK, Cohen JD (2001). An integrative theory of prefrontal cortex function. Annu Rev Neurosci 24: 167-202.

Muller-Dahlhaus JFM, Orekhov Y, Liu Y, Ziemann U (2008). Interindividual variability and age-dependency of motor cortical plasticity induced by paired associative stimulation. Exp Brain Res 187: 467-475.

Nishiyama M, Hong K, Mikoshiba K, Poo M, Kato K (2000). Calcium stores regulate the polarity and input specificity of synaptic modfication. Nature 408: 584-588.

Nitsche MA, Paulus W (2000). Excitability changes induced in the human motor cortex by weak transcranial direct current stimulation. J Physiol-London 527: 633-639.

Park JY, Jhung K, Lee J, An SK (2013). Theta-gamma coupling during a working memory task as compared to a simple vigilance task. Neurosci Lett 532: 39-43.

Percival DB, Walden AT (1993). Spectral Analysis for Physical Applications: Multitaper and Conventional Univariate Techniques. Cambridge University Press: Cambridge, MA, USA.

Petrides M, Pandya DN (1984). Projections to the frontal-cortex from the posterior parietal region in the rhesus-monkey. J Comp Neurol 228: 105-116.

Petrides M, Pandya DN (2002). Association pathways of the prefrontal cortex and functional observations. In: Stuss DTKnight RT (eds) Principles of Frontal Lobe Function. Oxford University Press: New York, NY, USA, pp 31-84.

Pignatelli M, Beyeler A, Leinekugel X (2012). Neural circuits underlying the generation of theta oscillations. J Physiol (Paris) 106: 81-92.

Potter-Nerger M, Fischer S, Mastroeni C, Groppa S, Deuschl G, Volkmann J et al (2009). Inducing homeostatic-like plasticity in human motor cortex through converging corticocortical inputs. J Neurophysiol 102: 3180-3190.

Ranganath C, D'Esposito M (2005). Directing the mind's eye: prefrontal, inferior and medial temporal mechanisms for visual working memory. Curr Opin Neurobiol 15: 175-182.

Rossi S, Hallett M, Rossini PM, Pascual-Leone A (2009). Safety, ethical considerations, and application guidelines for the use of transcranial magnetic stimulation in clinical practice and research. Clin Neurophysiol 120: 2008-2039.

Rothwell JC (1991). Physiological-studies of electric and magnetic stimulation of the human brain. Electroencephalogr Clin Neurophysiol 43: 29-35.

Rusjan PM, Barr MS, Farzan F, Arenovich T, Maller JJ, Fitzgerald $\mathrm{PB}$ et al (2010). Optimal transcranial magnetic stimulation coil placement for targeting the dorsolateral prefrontal cortex using novel magnetic resonance image-guided neuronavigation. Hum Brain Mapp 31: 1643-1652.

Stefan K, Kunesch E, Benecke R, Cohen LG, Classen J (2002). Mechanisms of enhancement of human motor cortex excitability induced by interventional paired associative stimulation. J Physiol-London 543: 699-708.

Stefan K, Kunesch E, Cohen LG, Benecke R, Classen J (2000). Induction of plasticity in the human motor cortex by paired associative stimulation. Brain 123: 572-584

Stefan K, Wycislo M, Classen J (2004). Modulation of associative human motor cortical plasticity by attention. J Neurophysiol 92: 66-72.

Tort ABL, Komorowski R, Eichenbaum H, Kopell N (2010). Measuring phase-amplitude coupling between neuronal oscillations of different frequencies. J Neurophysiol 104: 1195-1210.

Uhlhaas PJ, Singer W (2006). Neural synchrony in brain disorders: relevance for cognitive dysfunctions and pathophysiology. Neuron 52: 155-168.

Valeriani M, Restuccia D, Di Lazzaro V, Le Pera D, Barba C, Tonali $\mathrm{P}$ et al (1998). Dipolar sources of the early scalp somatosensory evoked potentials to upper limb stimulationeffect of increasing stimulus rates. Exp Brain Res 120: 306-315.

Valeriani M, Restuccia D, DiLazzaro V, Le Pera D, Tonali P (1997). The pathophysiology of giant SEPs in cortical myoclonus: a scalp topography and dipolar source modelling study. Evoked Potential-Electroencephalogr Clin Neurophysiol 104: 122-131.

Vaneden CG, Lamme VAF, Uylings HBM (1992). Heterotopic cortical afferents to the medial prefrontal cortex in the rat-a combined retrograde and anterograde tracer study. Eur J Neurosci 4: 77-97.

Voytek B, Canolty RT, Shestyuk A, Crone NE, Parvizi J, Knight RT (2010). Shifts in gamma phase-amplitude coupling frequency from theta to alpha over posterior cortex during visual tasks. Front Hum Neurosci 4: 9.

Ziemann U, Iliac TV, Pauli C, Meintzschel F, Ruge D (2004). Learning modifies subsequent induction of long-term potentiation-like and long-term depression-like plasticity in human motor cortex. J Neurosci 24: 1666-1672.

Zipser D, Kehoe B, Littlewort G, Fuster J (1993). A spiking network model of short-term active memory. J Neurosci 13: 3406-3420.

Supplementary Information accompanies the paper on the Neuropsychopharmacology website (http://www.nature.com/npp) 